patients who developed generalized tonic-clonic seizures (GTCS) and in those with the longest follow-up. In long-term follow-up of absence epilepsy (AE) patients, the incidence of GTCS was 50\%. Of those with AE and GTCS, 35\% were seizure free, whereas in the $50 \%$ with AE uncomplicated by GTCS, $78 \%$ were seizure free.

\title{
DRUG-INDUCED EXACERBATION OF BECTS
}

The incidence of drug-induced exacerbation of benign epilepsy with centrotemporal spikes (BECTS) was determined retrospectively in 82 patients examined at the Centre Saint Paul, Marseille, France. Among 40 patients treated with carbamazepine (35 monotherapy, 5 polytherapy) 1 showed electroclinical exacerbation that was dose related; discontinuation of CBZ was followed by immediate improvement. Among 14 taking phenobarbital ( 9 monotherapy, 5 polytherapy), 1 treated with CBZ and PB showed EEG exacerbation; the EEG improved after withdrawal of the PB while continuing CBZ. In 45 patients exposed to valproate (VPA) and 8 receiving benzodiazepine (BZP), none showed exacerbation. A spontaneous worsening of BECTS facilitated by the AED was considered a possibility. (Corda D, Gelisse P, Genton P et al. Incidence of druginduced aggravation in benign epilepsy with centrotemporal spikes. Epilepsia June 2001;42:754-759). (Reprints: Dr Pierre Genton, Centre Saint Paul, 13258 Marseille 09, France).

COMMENT. These authors found a minor risk of AED-exacerbation of BECTS. $\mathrm{CBZ}$ and phenobarbital were involved in only one case each, whereas VPA and BZP-treated patients showed no aggravation. Two previous reports of CBZexacerbation of BECTS are cited (Lerman, 1986; Caraballo et al, 1989).

An exacerbation of seizures and partial status epilepticus in 6 young, mentally retarded adults treated with $\mathrm{CBZ}$ polytherapy was related to excessive levels of CBZ-10, 11-epoxide, in a report from the Marshfield Clinic WI, and Mayo Clinic, MN. (So El et al, 1994; see Progress in Pediatric Neurology III, 1997;pp142143). CBZ-epoxide serum levels are important in CBZ-treated patients with seizure exacerbation.

Neuropsychological dysfunction and BECTS. Interictal epileptic discharges (IED) during sleep in patients with BECTS may be associated with impaired neuropsychological function. After spontaneous remission of the IED in 5 of 9 patients followed for 2 years, re-evaluation showed an increase in IQ scores and improvement in visuomotor coordination, memory, and attention. (Baglietto MG et al. Dev Med Child Neurol June 2001;43:407-412). The authors adocate further trials of high dose diazepam in short cycles to block interictal epileptic discharges in sleep in these patients.

\section{KETOGENIC DIET IN INFANTS WITH REFRACTORY EPILEPSY}

The effectiveness, tolerability, and adverse effects of the ketogenic diet were reviewed retrospectively in 31 infants (18 male; 13 female) with refractory epilepsy treated at Columbia-Presbyterian Medical Center, New York, NY. A 3:1 or 4:1 (fat:nonfat) ketogenic regimen was introduced slowly after a 12-38 hour inpatient fasting period to initiate ketosis. The ratio of the diet was adjusted to produce moderate to strong ketosis. Adequate calories and protein to sustain growth were provided. Mean age at start of diet was $13.8+/-5.7$ months; 14 were $<12$ months and $17>12$ months. The duration on the diet was $<3$ months in $3,3-5$ mo in 7 , and $=/>6$ mo in $21(67 \%)$. Epilepsy etiology was idiopathic in $12(39 \%)$ and symptomatic in $19(61 \%) ; 4$ patients had progressive metabolic and degenerative 
disorders. Seizure types were generalized in $10(32 \%)$, partial in 6, and mixed in 15 (48\%). Seventeen $(55 \%)$ had infantile spasms. Twenty four $(77 \%)$ were developmentally delayed.

Six patients (19\%) became seizure-free, $11(35 \%)$ were improved $(>50 \%$ decrease in frequency), and $14(45 \%)$ were not benefited. Antiepileptic medications were reduced concomitantly with improved dietary control. Patients with consistent moderate to severe ketonuria $(71 \%)$ showed a trend toward better seizure control than those with variable ketonuria (29\%). Level of ketonuria and level of seizure control were not significantly correlated. Favorable seizure control was increased sixfold in infants with infantile spasms; response was not related significantly to gender, developmental delay, or etiology. Parent questionnaires revealed that $75 \%$ noted a decreased frequency of seizures and $35 \%$ a lessening in seizure intensity. The majority reported improvements in the child's attention, alertness, activity level, and social interactions. An overall beneficial effect was reported by $50 \%$ of parents. Five infants developed adverse events that were reversible, including severe vomiting, renal stones, gastrointestinal bleeding, hyperlipidemia, and ulcerative colitis (the time on the diet before complication had been 7 to 26 months). One additional patient developed coma with hypoglycemia and acidosis after 8 days on the diet. Three patients died, 1 with a multisystem disease and pulmonary infection, 1 with aspiration, respiratory failure and congenital heart disease, and the third from complications related to an underlying pyruvate dehydrogenase deficiency. The authors recommend the ketogenic diet as safe and effective in infants with intractable epilepsy. (Nordli DR Jr, Kuroda MM, Carroll J et al. Experience with the ketogenic diet in infants. Pediatrics July 2001;108:129-133). (Reprints: Douglas $\mathbf{R}$ Nordli Jr MD, Epilepsy Center, Children's Memorial Hospital, Box 29, 2300 Children's Plaza, Chicago, IL 60614).

COMMENT. The ketogenic diet, if closely monitored by a major medical center team, can be an effective treatment in infants with refractory epilepsies, and especially for those with infantile spasms and myoclonic epilepsies. In addition, it is the recommended treatment for infants with pyruvate dehydrogenase deficiency (Wexler ID et al. Neurology 1997;49:1655-1661), and glucose transporter protein deficiency (DeVivo DC et al. N Engl I Med 1991;325:703709). Adverse events attributed to the diet are mainly reversible. These include nausea, vomiting and hypoglycemia, particularly prevalent during the initiation of the 4:1 ratio diet and with fasting, as recommended in the Johns Hopkins method. The omission of the period of fasting, and the introduction of ketosis using lesser fat/nonfat ratios, as practiced in the Mayo Clinic method, is efficacious and associated with fewer reports of adverse events (Millichap JG. Progress in Pediatric Neurology I, Chicago, PNB Publishers, 1991; pp84-88). Serious complications associated with high fat/nonfat ratios include renal stones, cardiomyopathy (Best et al, 2000; see Ped Neur Briefs July 2000;14:50), and platelet dysfunction with bleeding (Berry-Kravis E et al. 2001; see Ped Neur Briefs (Feb 2001;15:12-13). Serious adverse events were also reported in $10 \%$ of 52 children treated with a high 4:1 ratio/fat:nonfat/diet; complications included hypoproteinemia, lipemia, hemolytic anemia, renal tubular acidosis, and liver dysfunction with concomitant thrombocytopenia (Ballaban-Gil K et al. Epilepsia 1998;39:744-748). Despite the rigors and adverse events associated with the diet, in selected patients it can be a worthwhile method of treatment. Its effectiveness has been documented in numerous studies and reviews, from the initial report by Wilder RM (Mavo Clinic Bull 1921;2:307) to a recent confirmation in 150 children treated at Johns Hopkins University (Freeman JM et al. Pediatrics 1998;102:1358- 
1363). The present report is the first emphasizing the effectiveness of the ketogenic diet in infants. The attending adverse events and those reported in older children demonstrate the importance of close monitoring by a specialized medical team and support staff.

\section{LOW-DOSE PYRIDOXINE MASKS PYRIDOXINE-DEPENDENT SEIZURES}

A 4-month-old male infant with pyridoxine dependency and seizures from birth was controlled with extremely low-dose pyridoxine $(0.5 \mathrm{mg} /$ day $)$ given as a vitamin supplement, in a report from Joana de Gusmao Children's Hospital, Florianopolis, Brazil. Seizures were generalized myoclonic and tonic postures that began at 18 days of age and continued until 30 days. After a seizure-free period between 30 and 80 days, seizures returned and were refractory to antiepileptic drugs (AED) and prednisone. Examination showed global neurodevelopmental delay. An interictal EEG recorded continuous delta activity. MRI and laboratory tests were negative. Foliowing a diagnostic trial of pyridoxine $50 \mathrm{mg}$ twice daily by enteral tube, seizures ceased but the infant became comatose and hypotonic. After gradually regaining consciousness, the infant was discharged on $50 \mathrm{mg}$ pyridoxine orally, twice daily. Further history taking revealed that a multivitamin supplement containing $0.5 \mathrm{mg}$ pyridoxine daily had been given during the seizure-free interval between 30 and 80 days. An interruption in vitamin supplement coincided with the seizure relapse. At 2 years of age, the child had 2 brief seizures with fever. With an increase in pyridoxine dosage to 200 $\mathrm{mg} /$ day, no further seizures had occurred at last follow-up at 3 years, and development was normal. (Grillo E, da Silva RJM, Barbato JH Jr. Pyridoxinededpendent seizures responding to extremely low-dose pyridoxine. Dev Med Child Neurol June 2001;43:413-415). (Respond: Eugenio Grillo MD, Hospital Infantil Joana de Gusmao, Servico de Neurologia, Rua Rui Barbosa 152, 88025-301, Florianopolis SC, Brazil).

COMMENT. Low-dose pyridoxine given as a routine vitamin supplement may be sufficient to control seizures and mask an underlying pyridoxine-dependency. Early diagnosis and appropriate treatment may be delayed. In this case the masking dose of pyridoxine was very low, between 0.05 and $0,1 \mathrm{mg} / \mathrm{kg} / \mathrm{day}$.

Impaired consciousness and hypotonia have been reported in pyridoxine dependency after diagnostic doses given intravenously (Bankier et al, 1983), but rarely with enteral administration, as in this case. The associated irregular breathing was a concern, and further pyridoxine withdrawal and diagnostic testing were omitted.

Baxter P of the Sheffield Children's Hospital, UK, provides an excellent review of pyridoxine-dependent and -responsive seizures (Dev Med Child Neurol June 2001;43:416-420). Although rare, the varied modes of presentation of pyridoxine-dependent seizures result in misdiagnosis, particularly those with coincident hypoxic-ischemic encephalopathy, intraventricular hemorrhage, or hydrocephalus. Neuroimaging may show a variety of abnormalities, including paraventricular cysts, corpus callosum hypoplasia, cerebellar hypoplasia, white matter lesions, and hydrocephalus. Patients presenting after 1 month of age have a better outcome than those with earlier onset. Early and sustained treatment with adequate dosage influences outcome, especially in prevention or lessening of cognitive and developmental delays. With large doses of pyridoxine, monitoring of Romberg sign, ankle jerks, and joint position when possible is advised to check for sensory changes. There is no specific test for pyridoxine dependency, except for withdrawal and a further response to pyridoxine when seizures recur. As seen above, this test is not without hazard. Siblings and cousins may be affected and the 